\title{
ON A PROBLEM OF F. RIESZ CONCERNING PROXIMITY STRUCTURES
}

\author{
W. J. THRON
}

ABSTRACT. It is shown that every separated Lodato proximity is induced by the elementary proximity on a $T_{1}$ bicompactification of the original space.

A basic proximity structure $\Pi$ on a set $X$ is a relation on $\mathfrak{B}(X)$ satisfying the following requirements

$\mathrm{P}_{1}: \Pi=\Pi^{-1}$,

$\mathrm{P}_{2}: A \cup B \in \Pi(C) \Leftrightarrow A \in \Pi(C)$ or $B \in \Pi(C)$,

$\mathrm{P}_{3}: A \cap B \neq \varnothing \Rightarrow A \in \Pi(B)$,

$\mathrm{P}_{4}: \varnothing \notin \Pi(A) \forall A \in \mathfrak{P}(X)$.

Here $\Pi(A)=[B:\langle A, B\rangle \in \Pi]$. Each proximity structure induces a closure operator on $X$ as follows: $c_{n}(A)=[x:[x] \in \Pi(A)]$. If for a proximity relation the additional condition

$$
\mathrm{P}_{5}: c_{\pi}(A) \in \Pi(B) \Rightarrow A \in \Pi(B)
$$

also holds then $\Pi$ is called a $L O$-proximity. A relation $\Pi$ is said to be separated if in it

$$
\mathbf{P}_{6}:[x] \in \Pi([y]) \Leftrightarrow x=y,
$$

is valid.
A grill on $X$ is a family
$\mathrm{G}_{2}: A \cup B \in \mathfrak{G} \Rightarrow A \in \mathbb{F}$ or $B \in \mathbf{( 5}$,
$\mathrm{G}_{3}: \varnothing \notin \mathfrak{G}$.

Grills were introduced by Choquet [1] in 1947. It is known (see for example [5, Lemma 5.7]) that every grill is the union of ultrafilters. It is easy to verify that the converse also holds. It is an immediate consequence of $\mathrm{P}_{2}$ and $\mathrm{P}_{4}$ that $\Pi(A)$ is a grill for all $A \in \mathfrak{P}(X)$.

Define $b\left(\Pi,(\mathfrak{b})=\left[B: c_{\pi}(B) \in(\mathfrak{5}]\right.\right.$. One easily verifies that if $(\mathfrak{5}$ is a grill then $b\left(\Pi,(\mathfrak{5})\right.$ is a grill, $b\left(\Pi,(\mathfrak{H}) \supset\left(\mathfrak{F}\right.\right.$, and $\mathfrak{(}_{1} \supset\left(\mathfrak{F}_{2}\right.$ implies $b\left(\mathrm{II}, \mathfrak{G}_{1}\right) \supset$ $b\left(\Pi, \boldsymbol{G}_{2}\right)$.

Received by the editors December 13, 1972.

AMS (MOS) subject classifications (1970). Primary 54E05; Secondary 54D35.

Key words and phrases. Proximity, LO-proximity, elementary proximity, bunch, $\Pi$ II-clan, grill.

(c) American Mathematical Society 1973 
A grill $(5)$ for which it is true that $A, B \in \mathfrak{5} \Rightarrow A \in \Pi(B)$ will be called a $\Pi$-clan. A $\Pi$-clan (5) which satisfies the additional condition $b(\Pi, \mathfrak{5})=(\mathfrak{5}$ is called a bunch. by

Let $c$ be a Cech closure operator on $X$ then the relation $\Pi_{0}$ on $X$ defined

$$
A \in \Pi_{0}(B) \Leftrightarrow c(A) \cap c(B) \neq \varnothing
$$

is a basic proximity. It is called the elementary proximity associated with $c$. It is not in general true that $c=c_{\pi_{0}}$. However, if $\Pi$ is a separated $L O$ proximity then $c_{\pi}$ is the closure operator for a $T_{1}$-topology and if $c$ is a Kuratowski closure operator which generates a $T_{1}$-topology then $c=c_{\pi_{0}}$.

Let two proximity spaces $(X, \Pi)$ and $\left(Y, \Pi^{*}\right)$ and an injection $\varphi: X \rightarrow Y$ be given. Then $\Pi$ is said to be induced by $\Pi *$ if

$$
A \in \Pi(B) \Leftrightarrow \varphi(A) \in \Pi^{*}(\varphi(B)) .
$$

The problem of Riesz [6], referred to in the title, is the following: what types of proximity can be induced by elementary proximities on suitably constructed extension spaces of the original space? Riesz posed the problem in 1908, suggested a possible approach (using maximal $\Pi$-clans) but gave no answer. Clearly, the problem suggests that there may be a close relation between the proximities compatible with a given topological space and a certain class of topological extensions of the space. For $E F$-proximities Smirnov [7] in 1952 showed that they are induced by elementary proximities on $T_{2}$-bicompactifications of the underlying space. Improving on earlier work of Leader [3] and Lodato [4] Gagrat and Naimpally [2] recently showed that every separated $L O$-proximity which satisfies the additional condition:

$\mathrm{GN}$ : Given $A \in \Pi(B)$ there exists a bunch $\mathfrak{B}$ such that $A, B \in \mathfrak{B}$, is induced by the elementary proximity on a $T_{1}$-bicompactification of the original space.

We shall show that every $L O$-proximity satisfies GN (Theorem 4) and hence every separated $L O$-proximity can be induced by an elementary proximity. Harris has coined the name WI-proximity for those proximities which can be induced by an elementary proximity. He has shown that every separated $W I$-proximity is a $L O$-proximity. It now follows that the separated $W I$-proximities are exactly the separated $L O$-proximities.

The result stated above is the final link in a chain whose other members are also of interest.

THEOREM 1. Let $\mathfrak{F}$ be a filter and $\Pi$ a basic proximity on $X$; then $\Pi(\mathfrak{F})=\bigcap[\Pi(A): A \in \mathfrak{F}]$ is a grill. 
Proof. Clearly $\Pi(\mathfrak{F})$ satisfies conditions $\mathrm{G}_{1}$ and $\mathrm{G}_{3}$. Now assume $A \cup B \in \Pi(\mathfrak{F})$ and $A \notin \Pi(\mathfrak{F}), B \notin \Pi(\mathfrak{F})$. Then there exist sets $C$ and $D$ in $\mathfrak{F}$ such that $A \notin \Pi(C), B \notin \Pi(D)$. From this $A \notin \Pi(C \cap D), B \notin \Pi(C \cap D)$ follows. Since $C \cap D \in \mathfrak{F}$ we have $A \cup B \in \Pi(C \cap D)$ and thus a contradiction to the fact that $\Pi(C \cap D)$ is a grill.

THEOREM 2. Let $\Pi$ be a basic proximity on $X$ then $A \in \Pi(B)$ implies the existence of a П-clan (5) on $X$ such that $A, B \in \mathfrak{G}$.

Proof. Since $\Pi(B)$ is a grill it is a union of ultrafilters. Hence there exists an ultrafilter $\mathfrak{U}_{A}$ such that $A \in \mathfrak{U}_{A} \subset \Pi(B)$. It follows from the symmetry of $\Pi$ that $B \in \Pi\left(\mathfrak{U}_{A}\right)$. Since $\Pi\left(\mathfrak{U}_{A}\right)$ is a grill it follows that there exists an ultrafilter $\mathfrak{U}_{B}$ such that $B \in \mathfrak{U}_{B} \subset \Pi\left(\mathfrak{U}_{A}\right)$. Since $\mathfrak{U}_{B} \subset \Pi\left(\mathfrak{U}_{A}\right)$ implies $\mathfrak{U}_{A} \subset \Pi\left(\mathfrak{U}_{B}\right)$ a desired $\Pi$-clan is $\mathfrak{G}=\mathfrak{U}_{A} \cup \mathfrak{U}_{B}$.

THEOREM 3. Let $\Pi$ be a LO-proximity on $X$ then every maximal $\Pi$-clan is a bunch with respect to $\Pi$.

Proof. If $(\mathfrak{G}$ is a $\Pi$-clan than $b(\Pi, \mathfrak{G})$ is a $\Pi$-clan. To see this note that since $\Pi$ is a $L O$-proximity it satisfies $\mathrm{P}_{5}$ and hence $b(\Pi, \Pi(A))=\Pi(A)$. Since $\mathfrak{G}$ is a $\Pi$-clan we have $\mathfrak{b} \subset \Pi(A)$ for all $A \in \mathfrak{F}$. Hence $b(\Pi,(\mathfrak{5}) \subset$ $b(\Pi, \Pi(A))=\Pi(A)$. By symmetry of $\Pi, \mathfrak{5} \subset \Pi(B)$ for all $B \in b(\Pi, \mathfrak{b})$ and hence $b(\Pi,(\mathfrak{5}) \subset \Pi(B)$ for all $B \in b(\Pi, \mathfrak{5})$. It follows that $b(\Pi, \mathfrak{G})$ is a $\Pi$-clan. For every maximal $\Pi$-clan $\mathfrak{5}^{*}$ we then have $\mathfrak{5}^{*}=b\left(\Pi, \mathfrak{5}^{*}\right)$ (since $b(\Pi, \mathfrak{b}) \supset\left(\mathfrak{b}\right.$ for all grills $(\mathfrak{5})$. That is $\mathfrak{5}^{*}$ is a bunch.

THEOREM 4. Let $\Pi$ be a LO-proximity on $X$ and let $A \in \Pi(B)$. Then there exists a bunch $\mathfrak{B}$ containing $A$ and $B$.

Proof. Let $\mathfrak{G}$ be a $\Pi$-clan. There exists a maximal $\Pi I$-clan $\mathfrak{G}^{*}$ containing $\mathfrak{G}$. This is proved using Zorn's lemma. By Theorem $3 \mathfrak{G}^{*}$ is a bunch. By Theorem 2 a $\left(5\right.$ can be found to contain $A$ and $B$, hence $5^{*}$ contains the two sets.

A more extensive discussion of the ideas employed here is given in a forthcoming article [8] by the author.

\section{REFERENCES}

1. G. Choquet, Sur les notions de filtre et de grille, C.R. Acad. Sci. Paris 224 (1947), 171-173. MR 8, 333.

2. M. S. Gagrat and S. A. Naimpally, Proximity approach to extension problems, Fund. Math. 71 (1971), 63-76.

3. S. Leader, On clusters in proximity spaces, Fund. Math. 47 (1959), 205-213. MR 22 \#2978.

4. M. W. Lodato, On topologically induced general proximity relations, Proc. Amer. Math. Soc. 15 (1964), 417-422; II: Pacific J. Math. 17 (1966), 131-135. MR 28 \#4513; 33 \#695. 
5. S. A. Naimpally and B. D. Warrack, Proximity spaces, Cambridge Tracts in Math. Phys., no. 59, Cambridge Univ. Press, New York, 1970. MR 43 \#3992.

6. F. Riesz, Stetigkeitsbegriff und abstrakte Mengenlehre, Atti IV Congr. Internat. Mat. (Roma 1908), vol. 2, pp. 18-24.

7. Ju. M. Smirnov, On proximity spaces, Mat. Sb. (N.S.) 31 (73) (1952), 543-574; English transl., Amer. Math. Soc. Transl. (2) 38 (1964), 5-35. MR 14, 1107.

8. W. J. Thron, Proximity structures and grills, Math. Ann. (to appear).

Department of Mathematics, University of Colorado, Boulder, Colorado 80302 